\title{
A unique gel matrix moisturizer delivers deep hydration resulting in significant clinical improvement in radiance and texture
}

This article was published in the following Dove Press journal: Clinical, Cosmetic and Investigational Dermatology

\author{
Julie M Bianchini' \\ Qihong Zhang ${ }^{2}$ \\ Gabriel Hanna ${ }^{2}$ \\ Carol R Flach ${ }^{2}$ \\ Hequn Wang' \\ Michael D Southall' \\ Richard Mendelsohn ${ }^{2}$ \\ Manpreet Randhawa' \\ 'Johnson and Johnson Consumer \\ Companies, Inc., Skillman, NJ, USA; \\ ${ }^{2}$ Department of Chemistry, Rutgers \\ University, Newark, NJ, USA
}

\begin{abstract}
Introduction: As skin ages, it loses its ability to retain moisture and becomes rough and dry. This results in a clinically dull appearance with a loss of radiance, firmness, and suppleness. Symptoms can be improved with use of a moisturizer that builds and maintains skin hydration over time; however, most moisturizers that occlude the skin surface are perceived as heavy and greasy and are not consumer preferred.
\end{abstract}

Methods: A unique, consumer-preferred gel matrix formula was developed by combining liquid crystal structures, which mimic skin barrier lipid assembly, with specific emulsifiers that deliver water deep into skin. Ex vivo studies were conducted to investigate the superior hydrating effects of the gel matrix formula. Confocal Raman microscopy studies assessed the spatial distribution of water in ex vivo skin after application of the gel matrix formula. To determine the effects of the gel matrix formula on dry facial skin, a 12-week clinical study was conducted with subjects with self-perceived skin dryness and dullness.

Results: The formulation significantly increased the relative water content throughout epidermal regions, which was not observed with the application of a competitive gel formula. Instrumental measurements assessed improvements in skin surface moisturization and barrier function. Clinical grading showed significant improvements in hydration-related endpoints including radiance, clarity, and texture. Subject self-agree assessment demonstrated that subjects observed improvements in the appearance of their facial skin.

Conclusion: These studies demonstrated that the gel matrix formula increased skin water content in deeper layers, and resulted in significant clinical improvements in hydration, barrier function, and clinical appearance of radiance.

Keywords: confocal Raman microscopy, skin radiance, moisturization, dynamic skin barrier, skin hydration

\section{Introduction}

Hydration is essential for healthy skin, and many appearance benefits such as suppleness, smoothness, and radiance have been attributed to skin moisturization. ${ }^{1,2}$ The water content of a healthy stratum corneum (SC) is $20-30 \%{ }^{3}$, which can be negatively influenced by changes in barrier function, ${ }^{4,5}$ photodamage, ${ }^{6}$ as well as the molecular composition of the SC, including hygroscopic materials such as natural moisturizing factors. ${ }^{5}$ A decrease in skin hydration, which also correlates with increasing age, ${ }^{7,8}$ can result in a rough, dry, and clinically dull appearance with a loss of radiance, firmness, and suppleness. Increasing the water content of skin through therapeutic use of a moisturizer is an approach to improve the negative
Correspondence: Manpreet Randhawa Johnson \& Johnson Consumer

Companies, Inc., 199 Grandview Road,

Skillman, NJ 08558, USA

Phone + I 9089043062

Fax + I 9088741209

Email MRandhal@its.jnj.com 
clinical attributes of dry skin. However, traditional treatments for dry skin that consist of occlusive agents feel greasy to the touch, are poorly absorbed, and are not suitable for everyday use, and, therefore, result in poor compliance. $^{9}$ A therapeutic moisturizer that results in effective skin hydration and is delivered in a patient-preferred aesthetic formula could, therefore, provide a treatment for everyday use.

A unique, readily absorbable, gel matrix formula was developed with lightweight aesthetics to provide superior hydrating benefits while meeting patient demands for everyday use. This formula combines liquid crystal structures, which mimic the lipid assembly in the skin barrier, ${ }^{10}$ with specific emulsifiers and humectants. It is hypothesized that the gel matrix formula delivers deep skin hydration to improve the health and appearance of dry skin. The aim of this study was to evaluate whether treatment with the gel matrix formula delivered superior skin hydration compared to a leading competitive gel formula, as well as assess the clinical efficacy and consumer acceptability after longer periods of use (12 weeks).

Skin hydration status has been extensively evaluated using traditional biophysical measurement methodologies. Some of the broadly utilized methods include measurement of electrical conductance or the capacitance of the skin surface as an indicator of the skin surface hydration. ${ }^{11,12}$ Although conductance- and capacitance-based measurement tools, such as Skicon (IBS Co., Hamamatsu, Japan) and Corneometer ${ }^{\circledR}$ (CM 825, Courage + Khazaka electronic GmbH, Cologne, Germany), can be acquired quickly, they are indirect water measurements and cannot provide depth-resolved water content information. In addition, topical treatment ingredients can interfere with the readings, especially with salt-containing formulations. $^{13,14}$ Vibrational spectroscopy-based methods, such as confocal Raman microscopy (CRM), can directly measure $\mathrm{O}-\mathrm{H}$ vibrational modes providing direct measurements of relative water content inside the skin at different depths. ${ }^{15-17}$ Water concentration profiles of different body sites have been acquired using CRM, highlighting the validity of this measurement technique for analyzing skin hydration. ${ }^{7}$ In this study, the gel matrix formula was investigated and compared to a competitive gel formula using CRM to analyze the relative water content and its spatial distribution in ex vivo skin after topical application of the formulations.

This publication summarizes studies that investigated the water content after treatment with the gel matrix formula in different layers of epidermis in vitro, as well as the efficacy and moisturizing benefits of gel matrix formula through instrumental measurements and subject selfassessment in a human use clinical trial.

\section{Material and methods}

\section{Gel matrix formula}

A gel matrix facial moisturizer cream was developed which absorbs into skin quickly like a gel, with the longlasting moisturization of a cream. The formula contained glycerin, cetearyl olivate, sorbitan olivate, dimethicone, dimethicone/vinyl dimethicone crosspolymer, polyacrylamide, synthetic beeswax, C13-14 isoparaffin, dimethiconol, dimethicone crosspolymer, chlorphenesin, Laureth-7, carbomer, sodium hyaluronate, ethylhexylglycerin, C1214 Pareth-12, water, and phenoxyethanol. The formula contains glycerin and hyaluronic acid in the formula to provide humectancy. In addition, the formula contains a combination of ceteraryl olivate and sorbitan olivate, emulsifiers which have been reported to produce a liquid crystal structure within a formula that can capture water and other humectants (Hanno et al, 2015, and Park and An, 2007), whereas the presence of liquid crystals was confirmed by examining the formula under a polarizing microscope (Figure S1). Upon application to the skin, this gel matrix breaks apart to rapidly release water and humectants entrapped within the liquid crystals. This formula was specifically designed to utilize emulsifiers that mimic skin barrier lipids and to minimize the inclusion of oils or liquid esters commonly found in other moisturizers, which can produce undesirable greasy or oily aesthetics that can affect patient compliance.

\section{Ex vivo studies}

Ex vivo studies were performed using abdominal human skin explants obtained from normal human adults undergoing abdominoplasty surgeries. Informed consent was obtained from each patient, and the experimental protocol was approved by an Institutional Biosafety Committee. Skin sections were prepared under sterile conditions. Subcutaneous fat was carefully removed and then sections were flash frozen in liquid nitrogen and stored at $-80^{\circ} \mathrm{C}$. Skin samples were transported on dry ice to Rutgers University, Newark, NJ, where they were stored at $-20^{\circ} \mathrm{C}$ a maximum of 6 months prior to usage. For each experiment, skin samples were trimmed, defrosted, and the surface was cleaned with dry, followed by wet, cotton swabs. The skin was mounted in a Franz diffusion cell (FDC). For the untreated group, the skin surface was not modified further. For the treated groups, 20 
$\mu \mathrm{L}$ of either the gel matrix formula or the leading competitive gel formula was applied to the skin surface, and all samples were incubated at $34^{\circ} \mathrm{C}$ for $24 \mathrm{hrs}$ in a hydrated environment. The FDC was sealed with parafilm to maintain hydration, and the skin showed no visual signs of drying. Excess product was removed from the treated skin surface, and the sections were trimmed to fit into a homebuilt sample cell for the confocal Raman microscope. Details of the cell have been described previously. ${ }^{18}$ Briefly, the skin is sealed in the sample cell by pressing a glass coverslip on the surface, while hydration is maintained via a small water-filled well under the dermis. To avoid overhydration, special efforts were also made to make sure that the water in the hydration well during measurement was not touching the dermis. Six separate experiments were conducted for each treatment using a single donor.

\section{Confocal Raman microscopy and data analysis}

The sample cell was placed on the stage of the WITec Alpha-300R Plus confocal Raman microscope (Ulm, Germany). ${ }^{18}$ Briefly, a $532 \mathrm{~nm}$ laser was used for excitation and the sample was illuminated with a $100 \mathrm{X}$ oil immersion microscope objective (numerical aperture $=1.25$ ). The exposure power out of the objective was 20 $\mathrm{mW}$. A collection fiber (core diameter of $50 \mu \mathrm{m}$ ) was used to direct the Raman scattered light to the CCD detector, which gives a spatial resolution of $\sim 1-2 \mu \mathrm{m}$. Two image planes were acquired for each experiment with $5 \mathrm{~s}$ exposure time per spectrum. The scanning range was $80 \mu \mathrm{m}(X-$ axis) $\times 60 \mu \mathrm{m}$ (Z-axis) with a step size of $2 \mu \mathrm{m}$ for both axes. In total, 1,200 spectra were acquired for each image plane, and the spectral range was $200-3,800 \mathrm{~cm}^{-1}$.

Project FOUR 4.1 software (WITec) was used to process the confocal Raman data. Cosmic rays were removed (filter size of 4 and dynamic factor of 12) and the resulting data were baseline corrected using the Graph Background Subtraction Shape option with a shape size of 130 and noise factor of 1 . Cluster analysis ( $K$-means: without background, Euclidean normalization and distance mode, and an average of 4 clusters) was used to mask the top glass layer, thereby defining the skin surface. The relative water content was determined by obtaining the peak area ratio of the O-H stretching band $\left(3,084-3,675 \mathrm{~cm}^{-1}\right)$ to the Amide I band (1,640-1,678 $\left.\mathrm{cm}^{-1}\right)$. SC barrier strength was evaluated by taking the peak height ratio of a $\mathrm{C}-\mathrm{C}$ stretching band, indicative of all-trans acyl chains (ordered lipids,
$1,127 \mathrm{~cm}^{-1}$ ), to the phenylalanine ring breathing mode $\left(1,003 \mathrm{~cm}^{-1}\right)$. The Amide I and phenylalanine bands serve as internal standards to account for confocal losses in signal with depth. To delineate the boundary between the $\mathrm{SC}$ and the viable epidermis, the intensity ratio of the asymmetric $\mathrm{CH}_{2}\left(\sim 2,880 \mathrm{~cm}^{-1}\right)$ to the symmetric $\mathrm{CH}_{3}$ stretching band $\left(\sim 2,930 \mathrm{~cm}^{-1}\right)$ was used. This spectral parameter provides a measure of the relative lipid/protein content in skin, ${ }^{19}$ since the methylene stretching band arises predominantly from the lipid acyl chains and the methyl band is mostly due to the major epidermal protein, keratin. The known discontinuity in lipid content between the SC and VE is highlighted in Figure 1A, which displays images of the lipid/protein ratio. Specifically, the red regions reveal the high lipid/protein content of the $\mathrm{SC}$, whereas the green portraits the relatively lower lipid levels in the VE. In this study, the compressed intermediate region (yellow) was used to delineate the $\mathrm{SC}$ from the VE. Binary masks were created so that the water content in different skin regions could be evaluated. Nonparametric statistical analyses (Mann-Whitney rank sum tests, highest level of significance 0.001) were conducted using SigmaStat 3.5 and Sigma Plot 2,000. Mean values of the relative water content in the SC and VE were also calculated and compared among the various treatments.

\section{Clinical study design}

A single-center, full-face, 12-week clinical use study was conducted to assess the efficacy of the gel matrix formula compared to baseline. All subjects were female with selfperceived skin dryness and moderately dull skin between 35 and 55 years old, Fitzpatrick Skin Types I-III, and had a score between 3 and 6 on the face (on a $0-9$ scale with 0 being the best and 9 being the worse) at visit 1 for each of the following parameters, as determined by the principal investigator: skin radiance, skin tone evenness, visual firmness, and tactile firmness. Subjects with known allergies or sensitivities to topical products, any preexisting or dormant facial dermatologic condition, or visible hyperkeratinization were excluded from the study. Also subjects using over the counter products containing alpha-, beta-, or polyhydroxy acid, topical or oral retinoids 3 months prior to the study were excluded from the study as well. Prior to the study, all subjects were provided with an auxiliary cleanser and an auxiliary moisturizer to use in place of their regular facial cleansers, moisturizers, and antiaging products for a 7-day preconditioning period prior to 
A

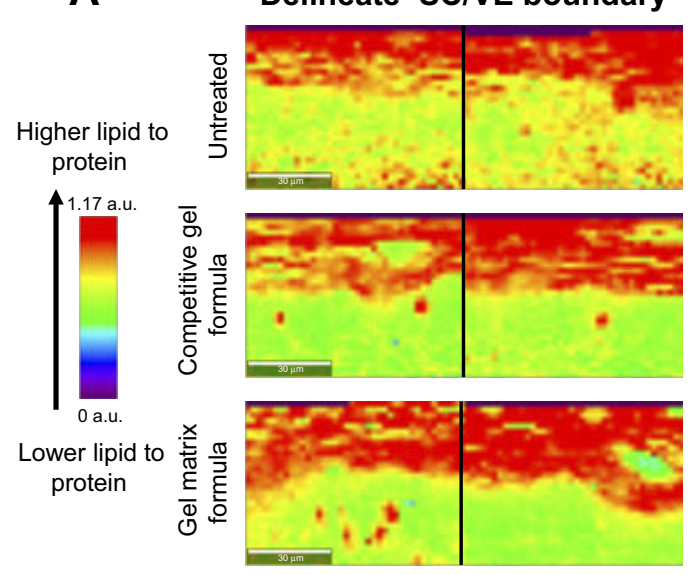

C

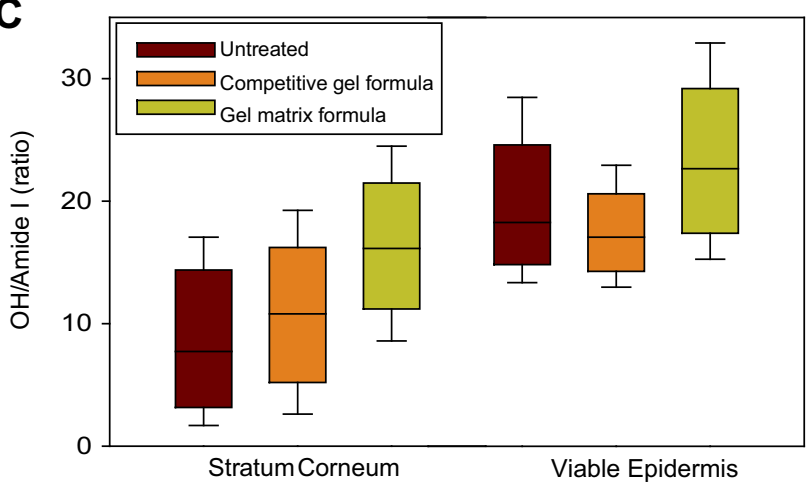

B
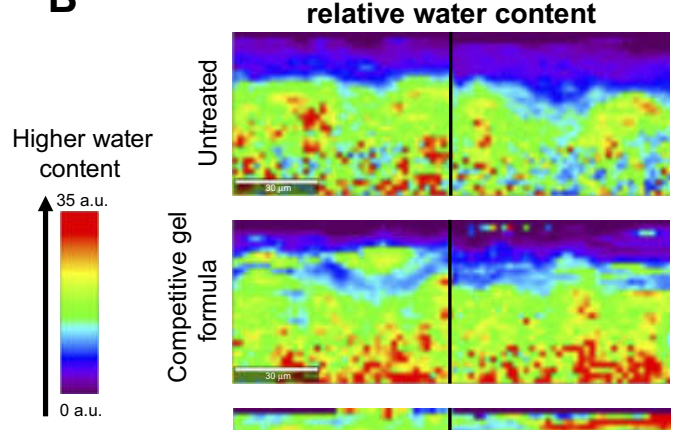

Lower water content
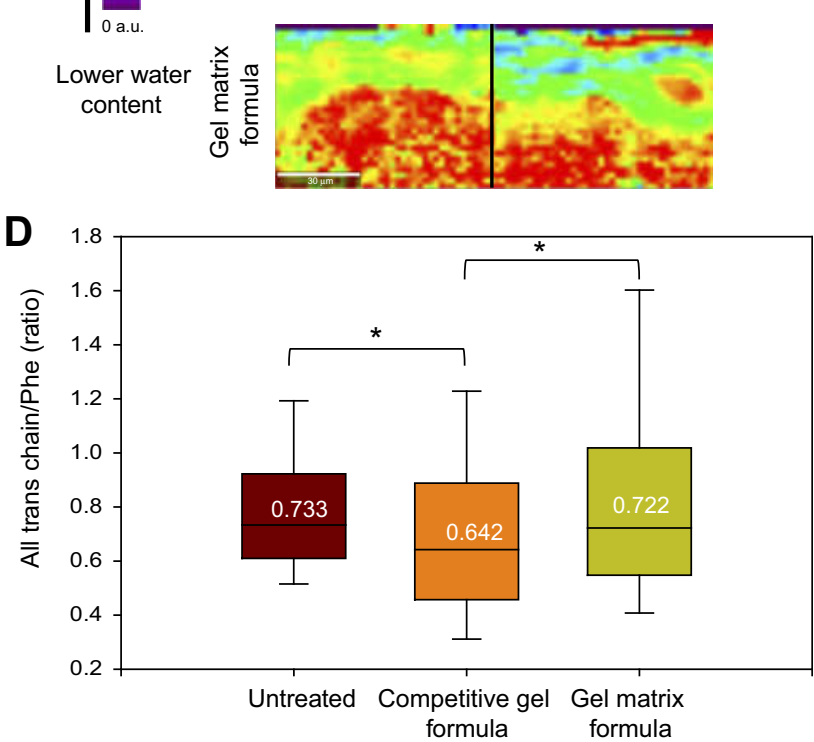

Figure I Ex vivo skin sample characterization using confocal Raman microscopy. (A) Images of the lipid/protein ratio delineating the SC/VE boundary (described in Methods section 2.2) for untreated, competitive gel formula, and gel matrix formula (top to bottom). Two images from the same experiment are shown side by side. Scale bar $=30$ microns. (B) Images of the relative water content with layout the same as in (A). (C) Box plots of relative water content in the SC and VE displaying 10th, 25th, median, 75th and 90th percentiles of six experiments (two images each) for untreated, competitive gel formula, and gel matrix formula treatments. (D) Box plots of the relative amount of ordered lipid in the SC (based on median values of six experiments as displayed in (C) for untreated, competitive gel formula, and gel matrix formula treatments. ${ }^{*} p \leq 0.05$.

baseline. At baseline, subjects were given the test gel matrix moisturizer to use for 12 weeks with twice-daily (morning/evening) application after washing their full face with the auxiliary cleanser. The test gel matrix moisturizer was applied to the full face, neck, and décolletage. Subjects were assessed at baseline and after 1 week, 4 weeks, 8 weeks, and 12 weeks of product use. Tolerance measures were evaluated using clinical evaluations (0-3 scale) of facial skin: expert evaluator grading of erythema, edema, scaling/peeling, and subject grading of burning/ stinging, itching, tightness.

The study was conducted in accordance to the Declaration of Helsinki principles. The study was approved by Allendale Investigational Review Board (Old Lyme, CT, USA), and the subjects' written informed consent was obtained prior to initiation of the investigation.

\section{Instrumental evaluation}

At each visit, the following measurements were taken on the subject's left cheek skin: transepidermal water loss (TEWL; via Tewameter, Courage-Khazaka Electronic $\mathrm{GmbH}$ ) and skin capacitance (via Corneometer, Courage-Khazaka Electronic $\mathrm{GmbH}$ ). For each instrument, replicate measurements were averaged for each time point, for each subject prior to statistical analysis.

\section{Clinical evaluation and questionnaires}

At each visit, the investigator assessed each subject's facial skin on a 0 (best) to 9 (worse) scale of facial skin for hydration-related endpoints including radiance, clarity, tactile texture, and visual texture. A subjective questionnaire made up of agree/disagree questions, and subjective attribute ratings were given to the subjects to complete at each visit after baseline. Subjects graded on 
a 1 to 5 scale, with 1 being disagree completely and 5 being agree completely.

\section{Statistical analysis}

Sample size of 30 completed subjects was determined based on the number of subjects typically used for antiaging phenotypic benefit-oriented study. The planned sample size of 30 completed subjects was determined based on the number of subjects typically used for antiaging phenotypic benefit-oriented studies. Also note that this sample size provides $>90 \%$ power to detect a population standardized effect size of 1 , where the standardized effect size is the mean change from baseline divided by the standard deviation for change from baseline. Historical data for similar studies have shown an estimated effect size of 1 or higher for measures such as skin capacitance, clarity, and texture at time points similar to this study.

For efficacy assessment, analysis of clinical evaluations was performed at week 1 and week 12. Paired $t$ tests were used to compare week 1 and week 12 vs baseline, based on a two-sided test at the 0.05 level of significance.

\section{Results}

\section{Topical treatment with the gel matrix formula increased water content in the stratum corneum and viable epidermis in ex vivo skin}

To investigate the potential superior hydrating benefits of the gel matrix formula, CRM was used to evaluate the relative water content and its spatial distribution in ex vivo skin after treatment (Figure 1). To quantify the differences between skin layers, the boundary of SC and VE was delineated using the lipid/protein ratio described in the "Materials and methods" section (see the "Ex vivo studies" section). Images of this parameter are shown in Figure 1A. All the images in Figure 1A and B were generated from skin samples that were either 1) untreated, 2) treated with the gel matrix formula, or 3) treated with a competitive gel formula. Two images per treatment condition are shown. All images have the same color scheme where red represents higher and purple represents lower water or lipid/protein content; thus, the intensities can be directly compared between the images for each parameter. Statistical analysis was conducted within each skin region to compare the treated samples to the untreated control, as well as the two treated samples to each other. Statistically significant differences $(p \leq 0.001)$ were found for all comparisons as are shown in the box plots in Figure 1C. Finally, Figure 1D shows the relative lipid conformational order of the treated samples compared to the untreated control sample.

Treatment with the gel matrix formula significantly increased the relative water content in the SC by $86 \%$ and in the VE by $18 \%$ as compared to the untreated control (based on mean values). In contrast, samples treated with a leading competitive gel formula only increased the water content in the SC by $34 \%$, which is less than half the increase observed in the sample treated with the gel matrix formula (Figure 1B). Furthermore, the water content of the VE in the sample treated with the leading competitive gel formula decreased by $11 \%$ compared to the untreated control. The superior hydrating effect of the gel matrix formula is further revealed when comparing the two treatment conditions to each other. Skin treated with the gel matrix formula showed an increase in the relative water content of the SC and VE layers by $39 \%$ and $33 \%$, respectively, compared to the leading competitive gel formula (Figure 1C).

The lipid conformational order of samples treated with the gel matrix formula was slightly changed compared to untreated samples $(p=0.020)$, while significantly lower lipid order was observed in samples treated with the leading competitive gel formula $(p \leq 0.001)$ (Figure 1D). The median value of the lipid conformational order parameter in the sample treated with the gel matrix formula closely resembles the untreated control sample. The sample treated with the leading competitive gel formula showed a significantly lower lipid conformational order compared to both the untreated control sample and the sample treated with the gel matrix formula. This result suggests that treatment with the gel matrix formula does not disrupt SC lipid order, while treatment with the leading competitive gel formula compromised SC barrier integrity.

Taken together, these results show that topical treatment of ex vivo skin with the gel matrix formula provides deep hydration resulting in increased water content, not only at the skin surface, but also throughout the VE to a depth of 60 microns. The two medians in the lipid order analysis are similar between this treatment and the untreated control condition suggests that the gel matrix formulation increases water content deep within the skin without causing barrier disorganization. 


\section{Topical treatment with the gel matrix} formula showed clinical improvements in skin surface hydration and barrier function

A total of 36 subjects were recruited, and 29 subjects completed the study. Of the 7 subjects that discontinued the study, 3 subjects requested withdraw, and 4 subjects failed to complete the follow up visits. No subjects discontinued use of gel matrix moisturizer due to irritation or discomfort. Subjects applied the gel matrix formula twice a day for 12 weeks, and corneometer and tewameter measurements were taken at the indicated end points (Figure 2).

Treatment with the gel matrix formula resulted in significantly improved corneometer measurements, which represents an increase in skin surface hydration, as soon as 1 week (Figure 2A). Because subjects were recruited with self-perceived dry skin, large and continual improvements in skin surface hydration were observed over the course of the study with over $75 \%$ of subjects showing improvement at each endpoint, and $89.3 \%$ of subjects showing improvement at week 12 (Figure 2B).

Treatment with the gel matrix formula is also hypothesized to enhance skin barrier function, because the gel matrix formula did not compromise lipid ordering as shown in Figure 1D. Continued use of the gel matrix formula over 12 weeks resulted in a significant decrease in TEWL measurements representing an improvement in skin barrier function (Figure 2C). The increase in barrier function measured at week 12 corresponded to $64.3 \%$ of subjects showing improvement compared to baseline (Figure 2D).

A

Corneometer Measurements

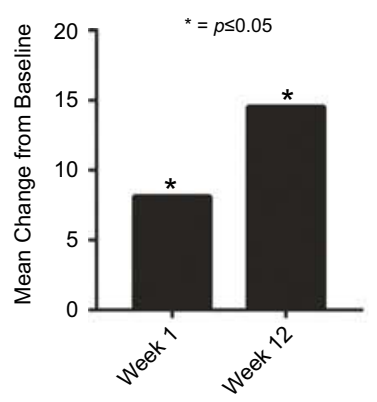

B

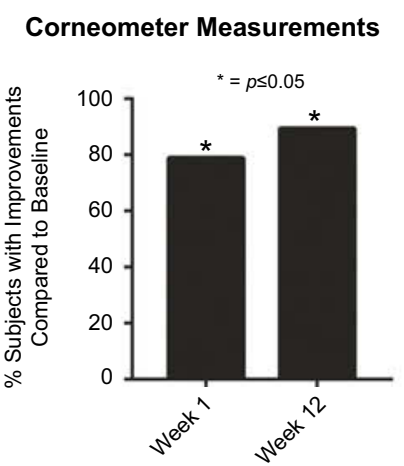

The results of the instrumental measurements demonstrated that treatment with the gel matrix formula led to a significant increase in skin hydration as soon as 1 week, and improvements in barrier function after 12 weeks.

\section{Treatment with the gel matrix formula showed significant improvements in clinical assessments of hydration related endpoints and resulted in a high percentage of subject self-agreement}

Subjects were graded for skin radiance, skin clarity, tactile texture and visual firmness (Figure 3 ) for clinical improvements in facial skin appearance. Throughout the study subjects showed significant improvement from baseline in all indicated efficacy parameters. No adverse effects related to the formulations were reported in the study. Improvements from baseline occurred as early as week 1, with continued improvements throughout the study (Figure $3 \mathrm{~A})$. After 12 weeks of application of the gel matrix formula, $100 \%$ of subjects showed improvements in skin radiance compared to baseline, $96.6 \%$ of subjects showed improvements in skin clarity, and the number of subjects that demonstrated improvements in tactile texture and visual texture were both $93.1 \%$ (Figure 3B). Furthermore, over half of the subjects showed improvements compared to baseline in the additional attributes of skin tone evenness and tactile firmness after 12 weeks of treatment (data not shown).

In contrast to traditional moisturizers that feel heavy and greasy and result in poor compliance, the gel matrix

C

Tewameter Measurements

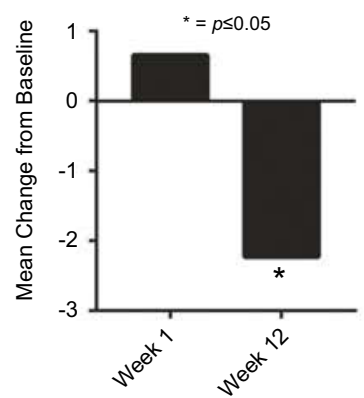

D

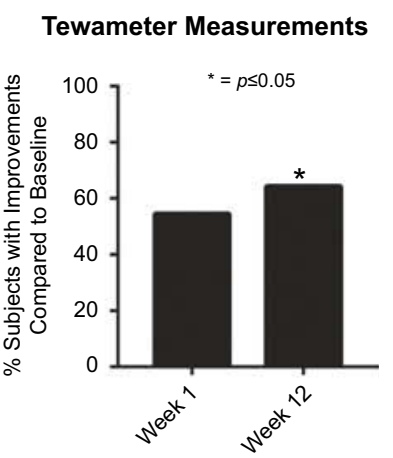

Figure 2 Treatment with the gel matrix formula significantly improved skin hydration and barrier function. (A) Paired $t$-test evaluation showed significant ( $p \leq 0.05$ ) improvements compared to baseline in corneometer measurements at week I and week 12 . (B) $78.8 \%$ of subjects showed improvements in surface skin hydration as soon as week I and $89.3 \%$ of subjects showed improvements by week I2. (C, D) Paired $t$-test evaluation showed significant ( $p \leq 0.05$ ) improvements compared to baseline in tewameter measurements at week 12 , which corresponded to a $64.3 \%$ of subjects showing improvements in barrier function. 

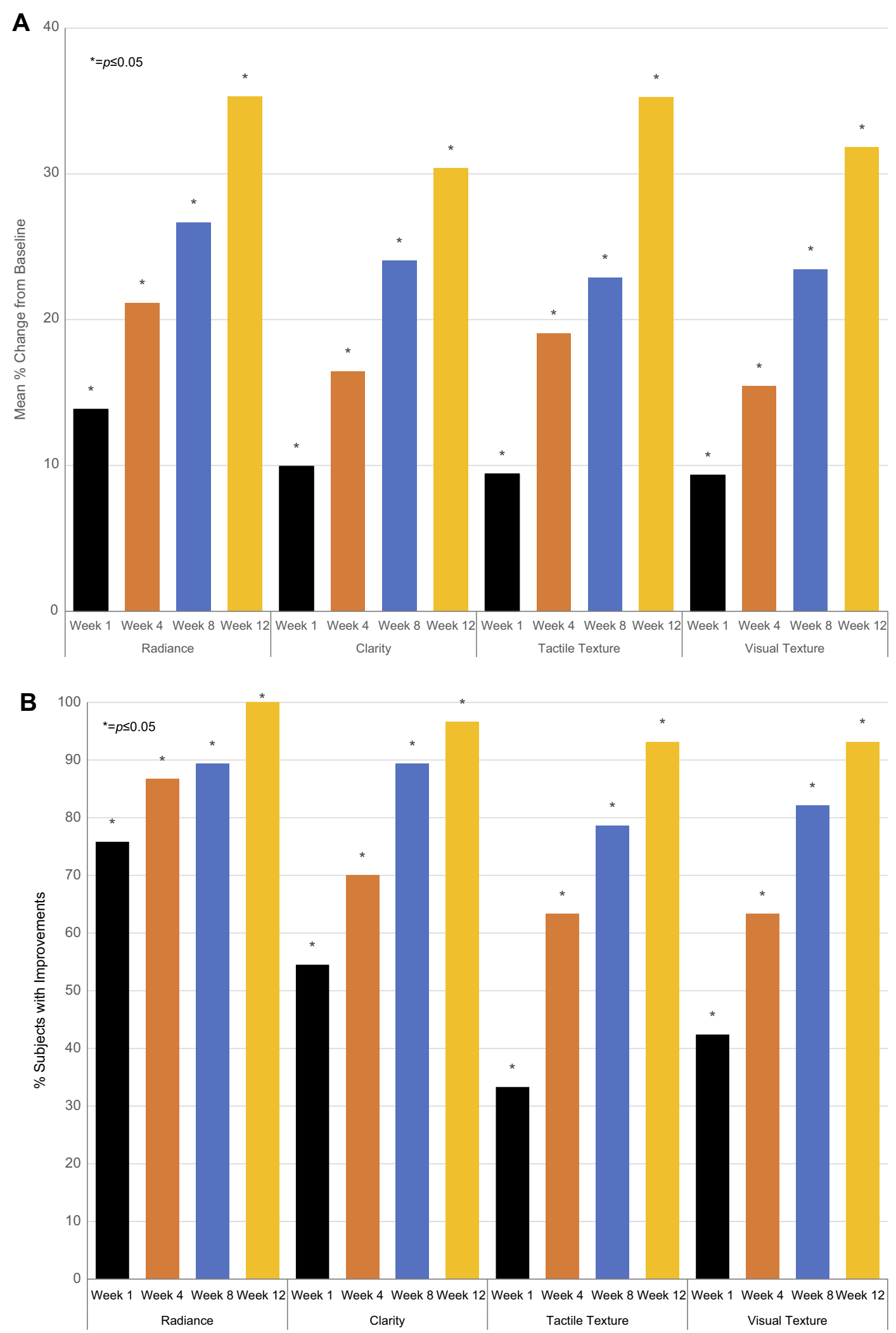

Figure 3 Treatment with the gel matrix formula significantly improved facial skin appearance. Dermatologist grading of hydration related endpoints: radiance, clarity, tactile texture, and visual texture. (A) Mean percent change from baseline, and (B) the percentage of subjects with improvements at each time point. Paired $t$-test evaluation showed significant $(p \leq 0.05)$ improvements compared to baseline for each efficacy parameter at all time points. 
Table I Treatment with the gel matrix formula represented a higher percentage of self-agree assessment at week 12. The percent of subjects that were in the top $2 \%$ agreement are shown for week I and week 12

\begin{tabular}{|c|c|c|}
\hline Self-Agree Assessment & $\begin{array}{l}\text { Week I } \\
\text { (Top 2\% } \\
\text { Agree) }\end{array}$ & $\begin{array}{l}\text { Week I } 2 \\
\text { (Top 2\% } \\
\text { Agree) }\end{array}$ \\
\hline $\begin{array}{l}\text { This product improves skin } \\
\text { tone and texture. }\end{array}$ & 63.6 & 75.9 \\
\hline $\begin{array}{l}\text { This product makes my skin } \\
\text { feel plump, firm, and resilient. }\end{array}$ & 57.6 & 69.0 \\
\hline $\begin{array}{l}\text { This product made my skin } \\
\text { look and feel smoother. }\end{array}$ & 78.8 & 82.8 \\
\hline $\begin{array}{l}\text { This product felt good on my } \\
\text { skin. }\end{array}$ & 81.8 & 89.7 \\
\hline $\begin{array}{l}\text { I feel the moisture penetrating } \\
\text { deep within my skin. }\end{array}$ & 60.6 & 69.0 \\
\hline $\begin{array}{l}\text { This product is an effective } \\
\text { moisturizer. }\end{array}$ & 72.7 & 79.3 \\
\hline
\end{tabular}

formula was designed to be aesthetically pleasing and suitable for everyday use. Therefore, it was essential to assess subject self-agreement towards improvements in clinical attributes, as well as formula favorability after continued use (Table 1). Positive results were obtained as soon as 1 week, with over half of the subjects in agreement about noticeable improvements in skin hydration and radiance related endpoints and favorable product aesthetics. Continued use of the gel matrix formula over 12 weeks resulted in over $75 \%$ of subjects in agreement with all statements. Notably, $82.8 \%$ of subjects agreed that the product made their skin look and feel smoother, and $79.3 \%$ of subjects agreed that this product is an effective moisturizer. In addition, after 12 weeks of treatment, subjects also agreed that they saw improvements in skin dullness/lack of radiance, the feeling of skin elasticity, the feeling of skin roughness, and the overall aged appearance (data not shown). These results demonstrate that the gel matrix formula is effective at delivering self-perceived improvements in facial skin appearance including specific improvements in skin hydration, tone, and texture, and is an aesthetically pleasing, effective moisturizer.

\section{Discussion}

A unique, consumer-preferred gel matrix formula was developed to deliver water deep into skin to provide immediate hydration, while also improving skin barrier function to lock in moisture. Ex vivo and clinical studies were conducted to investigate the superior hydrating effects of the gel matrix formula. Confocal Raman spectroscopy showed that treatment with the gel matrix formula provides deep hydration resulting from increased water content not only at the skin surface, but also throughout the layers of the VE, without causing barrier organization disruption. In comparison, a competitive gel formula failed to induce an increase in water content to the same extent and caused disorganization of the lipid barrier.

The superiority of the gel matrix formula compared to the competitive gel formula may be due to the fact that the gel matrix formula contains ingredients that form liquid crystal structures. The process of producing liquid crystals in a formula has been previously described (Hanno et al 2015, Park and An, 2007) and the liquid crystals are reported to help deliver water molecules deeper into the epidermis of the skin and result in a significant hydration benefit. In addition, the gel matrix formula does not contain alcohol, while the competitive gel formula does, which may have contributed to the observed lower lipid ordering in the sample treated with the competitive gel formula. Additional studies are required to determine whether the increase in water content observed after treatment with the gel matrix formula is delivered from the product, environment, or deeper dermal skin layers.

The clinical study demonstrated improved skin surface hydration as soon as 1 week, and improved barrier function after 12 weeks of treatment with the gel matrix formula. Furthermore, significant and continual improvements were observed from Dermatologist assessment of skin radiance, clarity, tactile texture, and visual texture. We hypothesize that treatment with the gel matrix formula resulted in clinical improvements in the appearance of facial skin radiance and youthfulness because increasing skin water content at both the surface and deeper layers improves the interaction between skin and light. Skin-light interaction occurs both at and beneath the skin surface. A small portion ( 4-7\%) of light is reflected off the SC surface, and most of the light ( $93-96 \%)$ penetrates deeper skin layers where it is back-scattered as subsurface reflected light. ${ }^{20,21}$ Hydrated skin has been proposed to enhance skin radiance by reducing surface roughness which promotes deeper penetration of incoming light (1). Surface roughness may be due to structural disorganization of the $\mathrm{SC}$ which can lead to optical heterogeneity between subsurface components. The resulting increase in back-scattered light from deeper dermal layers produces a diffused effect as light leaves the skin surface, which results in a more radiant appearance. ${ }^{1}$ Future work 
will better define the link between increased skin water content and perceived improvements in skin radiance and translucency.

Finally, in this study we assessed subject self-perceived improvements in facial skin appearance, as well as favorability of the gel matrix formula. The self-agree assessment demonstrated that treatment with the gel matrix formula made subjects' skin look and feel smoother. The majority of subjects agreed that the gel matrix formula felt good on their skin and is an effective moisturizer, which are important factors to ensure continued, every-day use.

The gel matrix formula has been demonstrated to deliver moisturization deep within the skin by utilizing unique ingredients that form liquid crystals within a gel matrix to mimic the chemical composition and possibly the physical organization of skin constituents. Unlike traditional therapeutic moisturizers, which work by occluding the skin surface by forming a hydrophobic barrier hence preventing water evaporation. The gel matrix formula also contains high amounts of water, hygroscopic humectants like hyaluronic acid that can attract water from both the dermal layers of skin and the surrounding environment, glycerol that promotes corneocyte resiliency, and emulsifiers that mimic skin barrier lipids. Finally, the gel matrix formula is light weight, aesthetically pleasing, and suitable for everyday consumer use, which has been confirmed by subject self-agree assessment.

\section{Conclusion}

Daily use of a therapeutic moisturizer is important for patients with dry, dull skin, which also occurs with aging. To help with compliance aesthetically pleasing gel matrix moisturizer was developed that can be used by subjects as part of their daily routine and that delivers both immediate hydration along with improving barrier function for longlasting benefits. The gel matrix formula with clinically proven benefits and consumer preferred aesthetics is an effective treatment for patients with dry and dull facial skin. Furthermore, robust clinical studies on cosmeceutical formulations provide excellent evidence-based results that enable practicing dermatologists to confidently recommend the most appropriate products for their patients.

\section{Acknowledgments}

The authors would like to thank David Lewin, PhD., Statistically Speaking Consulting, LLC for statistical analysis of data for this manuscript. Dr Lewin is a consultant for Johnson \& Johnson Consumer Companies, Inc.

\section{Disclosure}

Julie M Bianchini, Hequn Wang, Michael D Southall, and Manpreet Randhawa are employees of Johnson \& Johnson Consumer Companies, Inc., the manufacturer of the gel matrix moisturizer formulation tested. Qihong Zhang, Gabriel Hanna, Carol $\mathrm{R}$ Flach and Richard Mendelsohn are employees of Rutgers University, that received compensation for conducting the Confocal Raman Microscopy experiments. The preliminary findings from this paper were presented at the 2017 American Academy of Dermatology as a poster presentation. Dr Carol R Flach report grants from Johnson and Johnson Consumer Companies, Inc., during the conduct of the study. Dr Richard Mendelsohn report grants from J\&J Consumer Companies, Inc., during the conduct of the study.

\section{References}

1. Jiang ZX, DeLaCruz J. Appearance benefits of skin moisturization. Skin Res Technol. 2011;17(1):51-55.

2. Rawlings J. Skin Moisturization. New York: Macerl Dekker; 2002.

3. Draelos ZD. Concepts in skin care maintenance. Cutis. 2005;76(6 Suppl):19-25.

4. Rawlings AV, Harding CR. Moisturization and skin barrier function Dermatol Ther. 2004;17(Suppl 1):43-48.

5. Rawlings AV, Matts PJ. Stratum corneum moisturization at the molecular level: an update in relation to the dry skin cycle. $J$ Invest Dermatol. 2005;124(6):1099-1110. doi:10.1111/j.15231747.2005.23726.x

6. Wan M, Hu R, Xie X, et al. Skin erythema, pigmentation and hydration kinetics after ultraviolet radiation-induced photodamage in southern Chinese women. Photochem Photobiol. 2017;93(5):12761281. doi:10.1111/php.2017.93.issue-5

7. Egawa M, Tagami H. Comparison of the depth profiles of water and water-binding substances in the stratum corneum determined in vivo by Raman spectroscopy between the cheek and volar forearm skin: effects of age, seasonal changes and artificial forced hydration. $\mathrm{Br} J$ Dermatol. 2008;158(2):251-260. doi:10.1111/j.13652133.2007.08311.x

8. Potts RO, Buras EM Jr., Chrisman DA Jr. Changes with age in the moisture content of human skin. J Invest Dermatol. 1984;82(1):97100.

9. Sethi A, Kaur T, Malhotra SK, Gambhir ML. Moisturizers: the slippery road. Indian J Dermatol. 2016;61(3):279-287. doi:10.4103/ 0019-5154.182427

10. Olivem 1000 Crystal Skin. The First Emulsifying Active Ingredient. Milan, Italy: The B\&T Company; 2008.

11. Tagami H. Quantitative measurements of water concentration of the stratum corneum in vivo by high-frequency current. Acta Derm Venereol Suppl (Stockh). 1994;185:29-33.

12. Tagami H, Kanamaru Y, Inoue K, et al. Water sorption-desorption test of the skin in vivo for functional assessment of the stratum corneum. J Invest Dermatol. 1982;78(5):425-428.

13. Li F, Conroy E, Visscher M, Wickett RR. The ability of electrical measurements to predict skin moisturization. I. Effects of $\mathrm{NaCl}$ and glycerin on short-term measurements. J Cosmet Sci. 2001;52(1):13-22.

14. Dykes PJ. What are meters measuring? Int J Cosmet Sci. 2002;24 (4):241-245. doi:10.1046/j.1467-2494.2002.00146.x 
15. Verzeaux L, Vyumvuhore R, Boudier D, et al. Atopic skin: in vivo Raman identification of global molecular signature, a comparative study with healthy skin. Exp Dermatol. 2018;27:403-408.

16. Hoppel M, Kwizda K, Baurecht D, Caneri M, Valenta C. The effect of a damaged skin barrier on percutaneous absorption of SDS and skin hydration investigated by confocal Raman spectroscopy. Exp Dermatol. 2016;25(5):390-392. doi:10.1111/exd.12950.

17. Forster M, Bolzinger MA, Montagnac G, Briancon S. Confocal Raman microspectroscopy of the skin. Eur J Dermatol. 2011;21 (6):851-863

18. Pyatski Y, Zhang Q, Mendelsohn R, Flach CR. Effects of permeation enhancers on flufenamic acid delivery in Ex vivo human skin by confocal Raman microscopy. Int J Pharm. 2016;505(1-2):319-328. doi:10.1016/j.ijpharm.2016.04.011
19. Janssens M, van Smeden J, Puppels GJ, Lavrijsen AP, Caspers PJ, Bouwstra JA. Lipid to protein ratio plays an important role in the skin barrier function in patients with atopic eczema. $\mathrm{Br} J$ Dermatol. 2014;170(6):1248-1255. doi:10.1111/bjd.2014.170. issue-6

20. Matsubara A. Differences in the surface and subsurface reflection characteristics of facial skin by age group. Skin Res Technol. 2012;18 (1):29-35. doi:10.1111/srt.2011.18.issue-1

21. Lister T, Wright PA, Chappell PH. Optical properties of human skin. J Biomed Opt. 2012;17(9):90901. 


\section{Supplementary material}

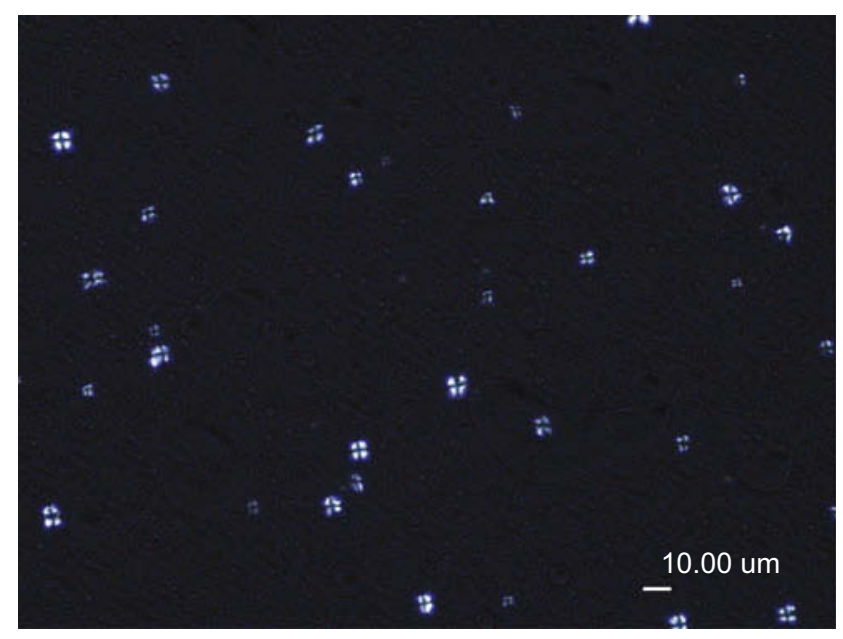

Figure SI Presence of liquid crystals in gel formulation. The presence of liquid crystals in the gel cream formulation was confirmed by assessing the final formula under a polarizing microscope.

\section{Publish your work in this journal}

Clinical, Cosmetic and Investigational Dermatology is an international, peer-reviewed, open access, online journal that focuses on the latest clinical and experimental research in all aspects of skin disease and cosmetic interventions. This journal is indexed on CAS.
The manuscript management system is completely online and includes a very quick and fair peer-review system, which is all easy to use. Visit http://www.dovepress.com/testimonials.php to read real quotes from published authors. 